
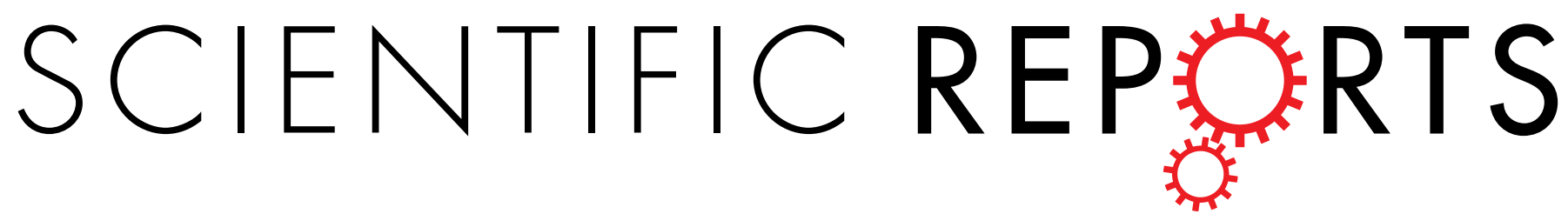

\title{
OPEN Electronic transport properties of Ir-decorated graphene
}

Received: 24 July 2015

Accepted: 28 September 2015

Published: 28 October 2015
Yilin Wang ${ }^{1,2,4}$, Shudong $\mathrm{Xiao}^{2, *}$, Xinghan $\mathrm{Cai}^{2,+}$, Wenzhong Bao ${ }^{2,5}$, Janice Reutt-Robey ${ }^{4}$ \& Michael S. Fuhrer ${ }^{1,2,3}$

Graphene decorated with $5 d$ transitional metal atoms is predicted to exhibit many intriguing properties; for example iridium adatoms are proposed to induce a substantial topological gap in graphene. We extensively investigated the conductivity of single-layer graphene decorated with iridium deposited in ultra-high vacuum at low temperature $(7 \mathrm{~K})$ as a function of Ir concentration, carrier density, temperature, and annealing conditions. Our results are consistent with the formation of Ir clusters of $\mathbf{1 0 0}$ atoms at low temperature, with each cluster donating a single electronic charge to graphene. Annealing graphene increases the cluster size, reducing the doping and increasing the mobility. We do not observe any sign of an energy gap induced by spin-orbit coupling, possibly due to the clustering of Ir.

Graphene, a two-dimensional honeycomb structure of carbon atoms, has been intensively studied due to its novel electronic and structural properties ${ }^{1}$. A striking aspect of graphene is that every atom is a surface atom, and the two-dimensional electron gas in graphene is exposed at the surface. This allows graphene's electronic properties to be tuned by the appropriate introduction of disorder/impurities, such as vacancies ${ }^{2}$, adatoms $s^{3-7}$ and various molecules ${ }^{8-10}$. For example, physisorbed potassium donates an electron to graphene and the ions act as charged impurity scattering centers, decreasing the mobility and conductivity of graphene $e^{3}$; while chemisorbed hydrogen and fluorine introduce resonant scattering centers, inducing a band gap and insulating behavior ${ }^{11,12}$. Recently, transition metal adatoms on graphene have attracted great attention due to a number of fascinating theoretical predictions ${ }^{13-15}$. Several $5 d$ metal atoms are expected to induce the quantum spin Hall effect ${ }^{13}$ or quantum anomalous Hall effect ${ }^{14}$ in graphene due to the enhanced spin-orbit coupling in graphene. Graphene decorated with iridium (Ir) or osmium adatoms is predicted to realize a two-dimensional topological insulator protected by a substantial band gap $(\sim 300 \mathrm{meV})^{15}$. These predictions motivate the experimental study of the properties of graphene decorated with $5 d$ heavy metals ${ }^{16-19}$.

In this work, we investigate the in-situ transport properties of single-layer graphene decorated with Ir deposited at low temperature $(7 \mathrm{~K})$ under ultra-high vacuum (UHV) conditions. We measure the conductivity as a function of Ir concentration, carrier density, temperature, and annealing conditions. The results are consistent with the formation of clusters of Ir on graphene, even for deposition at low temperature, with each cluster containing $\sim 100 \mathrm{Ir}$ atoms and donating $\sim 1$ electron to graphene and acting as a charged impurity scattering center. Annealing Ir-decorated graphene to room temperature greatly reduces the doping and increases the mobility, consistent with greatly increased cluster size. No signature of any significant bandgap in graphene decorated with Ir adatoms was observed and is attributed to the formation of Ir clusters.
${ }^{1}$ Department of Physics, University of Maryland, College Park, MD 20742, USA. ${ }^{2}$ Center for Nanophysics and Advanced Materials, University of Maryland, College Park, MD 20742, USA. ${ }^{3}$ School of Physics, Monash University, Victoria 3800, Australia. ${ }^{4}$ Department of Chemistry and Biochemistry, University of Maryland, College Park, MD 20742, USA. *Present address: Department of Electrical Engineering, University of Notre Dame, Notre Dame, Indiana 46556, USA. 'Present address: Department of Physics, University of Washington, Seattle, Washington 98195, USA. SPresent address: State Key Laboratory of ASIC and System, Department of Microelectronics, Fudan University, Shanghai 200433, China. Correspondence and requests for materials should be addressed to M.S.F. (email: michael.fuhrer@monash.edu) 

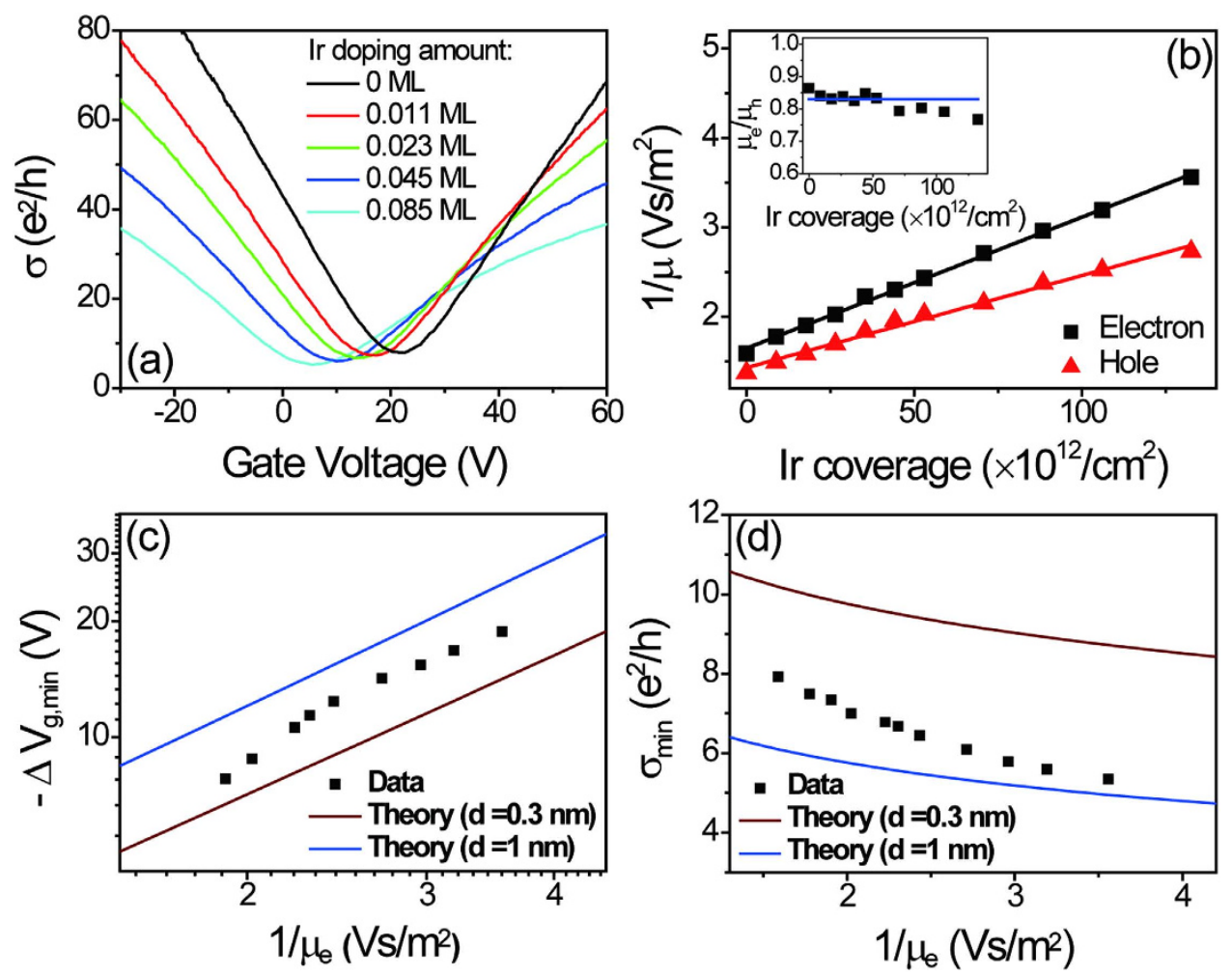

Figure 1. Conductivity evolution after Ir deposition. (a) The conductivity $\sigma$ versus gate voltage $V_{g}$ for pristine graphene and at four different Ir coverages taken at $7 \mathrm{~K}$. Here $1 \mathrm{ML}=1.56 \times 10^{15} \mathrm{~cm}^{-2}$ is defined from the atomic density of the $\operatorname{Ir}(111)$ surface. (b) Inverse of electron mobility $1 / \mu_{\mathrm{e}}$ and hole mobility $1 / \mu_{\mathrm{h}}$ versus Ir coverage. Lines are linear fits to all data points. Inset: The ratio of $\mu_{\mathrm{e}}$ to $\mu_{\mathrm{h}}$ versus Ir coverage. The blue line corresponds to the electron-hole asymmetry observed for potassium in ref. 3. (c) The shift of gate voltage of minimum conductivity $-\Delta V_{g, \min }$ as a function of $1 / \mu_{\mathrm{e}}$, which is proportional to the impurity concentration. All $-\Delta V_{g, \min }$ values are offset by $2 \mathrm{~V}$ to account for initial disorder. (d) The minimum conductivity $\sigma_{\min }$ as a function of $1 / \mu_{\mathrm{e}}$. Lines in $(\mathbf{c}, \mathbf{d})$ correspond to the theory in ref. 3 .

\section{Results}

Conductivity of as-fabricated and Ir-decorated graphene. Ir was deposited via electron-beam evaporation in UHV. To vary the coverage, the device was exposed to a controlled flux with sequential exposures at a fixed sample temperature of $7 \mathrm{~K}$. Following each deposition, the conductivity as a function of gate voltage $\sigma\left(\mathrm{V}_{\mathrm{g}}\right)$ was measured. Figure 1(a) shows $\sigma\left(\mathrm{V}_{\mathrm{g}}\right)$ for the pristine device and the device with four different Ir doping concentrations. With increasing Ir deposition, several features become apparent: 1) the gate voltage of minimum conductivity $V_{g, m i n}$ shifts to more negative values, 2) the mobility $\mu$ decreases, 3 ) the minimum conductivity $\sigma_{\min }$ decreases. All of these features are similar to the effect of charged impurities on graphene, observed previously by deposition of potassium ${ }^{3}$; we will discuss each in detail below.

\section{Discussion}

We fit $\sigma\left(V_{\mathrm{g}}\right)$ at high $\left|V_{\mathrm{g}}\right|$ to

$$
\sigma\left(V_{g}\right)=n e \mu=\mu c_{g}\left(V_{g}-V_{g, \min }\right)+\sigma_{r e s}
$$

separately for electron conduction $\left(V_{\mathrm{g}}-V_{\mathrm{g}, \min }>0\right)$ and hole conduction $\left(V_{\mathrm{g}}-V_{\mathrm{g}, \min }<0\right)$ in order to determine the electron and hole mobilities $\mu_{e}$ and $\mu_{h}$, the threshold shift $\Delta V_{\mathrm{g} \text {,min }}$, and the residual conductivity $\sigma_{\text {res }}$, where $n$ is the carrier density, $e$ is the electronic charge and $c_{\mathrm{g}}$ is the gate capacitance per area. Figure 1 (b) shows inverse of electron mobility $1 / \mu_{\mathrm{e}}$ and hole mobility $1 / \mu_{\mathrm{h}}$ versus Ir coverage, both of which are linear, demonstrating the mobility depends inversely on the density of impurities $1 / \mu \propto$ $n_{\text {imp }}$ (Matthiessen's rule $)^{20}$ :

$$
\sigma(n)=C e\left|\frac{n}{n_{\text {imp }}}\right|
$$




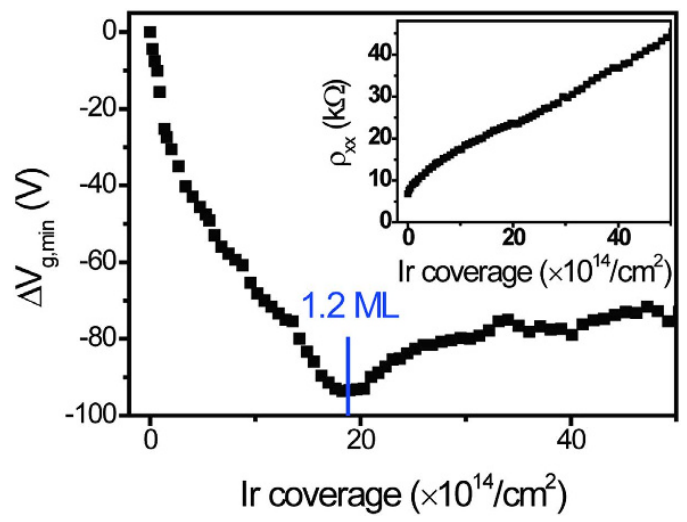

Figure 2. Ir coverage dependence of the shift of gate voltage of minimum conductivity of graphene $\Delta V_{g, \text { min }}$. Inset: The resistivity of graphene at $V_{g}=V_{g, \text { min }}$ as a function of Ir coverage. The measurement was carried out during the continuous deposition of Ir.

where $C$ is a constant. Although the $\mu_{\mathrm{e}}$ and $\mu_{\mathrm{h}}$ are distinct, their ratio $\mu_{\mathrm{e}} / \mu_{\mathrm{h}}$ remains approximately 0.8 before and with increasing Ir coverage, as shown in the inset of Fig. 1(b). The similar electron-hole asymmetry in mobility is also observed for scattering by potassium adsorbates ${ }^{3}$ and follows from the electrostatic environment of the graphene sample ${ }^{21}$. The constant $C$ is $7 \times 10^{17} \mathrm{~V}^{-1} \mathrm{~s}^{-1}\left(9 \times 10^{17} \mathrm{~V}^{-1} \mathrm{~s}^{-1}\right)$ for electrons (holes), about two orders of magnitude larger than found for $\mathrm{K}$ adatoms, indicating $\mathrm{Ir}$ is about 2 order of magnitude less effective at scattering electrons in graphene. Figure $1(\mathrm{c}, \mathrm{d})$ show $\Delta \mathrm{V}_{\mathrm{g} \text {,min }}$ and $\sigma_{\min }$ as a function of $1 / \mu_{\mathrm{e}}$, respectively. The results of both $\Delta \mathrm{V}_{\mathrm{g} \text {,min }}$ and $\sigma_{\min }$ agree well with that of potassium adatoms and can be well described by the previous theoretical predictions generated for impurity charge $Z e$ with $Z=1$, and impurity-graphene distance $d=0.3 \mathrm{~nm}-1.0 \mathrm{~nm}^{3,20}$. Notably, the theoretical predictions are very different for $Z \neq 1$. For example, for a fixed charge transfer $\Delta V_{\mathrm{g}, \min }=Z n_{\text {imp }}$, the scattering cross-section of an impurity scales as $Z^{2}$ however the density of impurities $n_{\text {imp }}$ scales as $1 / Z$, hence the mobility scales as $Z$. Thus the results strongly suggest scattering by charged impurities with $\mathrm{Z} \approx 1$. Together with the observation of $C$ about two orders of magnitude larger for Ir adatoms than for $\mathrm{K}$ adatoms, we infer that scattering is due to clusters ${ }^{22}$ of around $100 \mathrm{Ir}$ adatoms with a total charge of $\sim 1 \mathrm{e}$. This is entirely consistent with the observation of $\Delta V_{\mathrm{g}, \min }$ about two orders of magnitude lower at a given Ir concentration than for a similar concentration of $\mathrm{K}$ adataoms. Note also that the charge transferred by Ir in clusters is much smaller than the value for isolated Ir adatoms calculated by density functional theory $(Z=0.22)^{23}$. It is somewhat surprising that Ir forms clusters of this size so readily on a graphene substrate of $T=7 \mathrm{~K}$. However the calculated barrier for an $\mathrm{Ir}$ adatom to diffuse through the bridge site on graphene is very small, $\sim 50 \mathrm{meV}^{15}$ and the $\mathrm{Ir}$ - Ir binding is stronger than the Ir-C binding ${ }^{22}$, therefore Ir adatoms are highly mobile on graphene and susceptible to form three dimensional clusters even at low temperature.

We also explored very high Ir coverages $(>1 \mathrm{ML})$. Figure 2 shows the shift of $V_{\mathrm{g}, \min }$ as a function of Ir coverage; $\sigma\left(\mathrm{V}_{\mathrm{g}}\right)$ was measured during the continuous deposition of Ir. At the beginning of deposition, Ir tends to form uniform clusters randomly distributed on the graphene surface, and $V_{\mathrm{g}, \mathrm{min}}$ drops fast and is roughly linear with increasing Ir coverage. At higher coverages $V_{g \text {, min }}$ drops at a slower rate, consistent with the formation of larger clusters, and finally reaches a saturated value at the Ir coverage of $1.2 \mathrm{ML}$. This presumably marks the transition from clusters to a continuous film. With increased coverage beyond this point, $V_{g, \min }$ gradually recovers. The results are similar to those obtained for $\mathrm{Pt}^{5}$, where it was also observed that small clusters produced $n$-type doping, with a reduction or even reversal of doping as a continuous film is formed. Monolayer graphene on single crystal Ir is known to be slightly $p$-doped. We conclude that the larger the cluster size, the smaller the charge transfer efficiency. Although $V_{\mathrm{g} \text {, min }}$ begins to recover for coverages above 1.2 ML, the resistivity at the Dirac point $\left(\rho_{\mathrm{xx}}\right)$ continues to rise with increasing Ir coverage, as shown in the inset of Fig. 2. This indicates the failure of charged-impurity scattering to describe the high coverage regime. Presumably other types of disorder, potentially short ranged scattering, also play an important role in this regime, but this requires additional work to understand.

We also explored temperature as a means to tune the cluster size after deposition ${ }^{4}$. Figure 3(a) shows $V_{\mathrm{g}, \min }$ of graphene with $0.085 \mathrm{ML}$ Ir deposited at $T=7 \mathrm{~K}\left[\sigma\left(\mathrm{V}_{\mathrm{g}}\right)\right.$ data shown in Fig. 1(a) $]$ as a function of temperature. During warming, $V_{\mathrm{g} \text {,min }}$ first shifts slightly towards negative gate voltage and then for $T>90 \mathrm{~K}$ shifts more rapidly to positive, eventually reaching its initial value before $\mathrm{Ir}$ deposition. The $V_{\mathrm{g}}$, min shift reflects the rearrangement of Ir clusters, while the diffusion, growth and nucleation of atoms on the surface is a complex process, we speculate that below $90 \mathrm{~K}$, Ir clusters do not grow appreciably, while some movement of individual adatoms leads to the negative gate voltage shift of $V_{\mathrm{g} \text {,min }}$. Above $90 \mathrm{~K}$, Ir clusters grow by Ostwald ripening, reducing the charge transfer efficiency and positively shifting $V_{g, \min }$. Unsurprisingly, formation of large clusters at higher temperature $(350 \mathrm{~K})$ is found to be irreversible when 

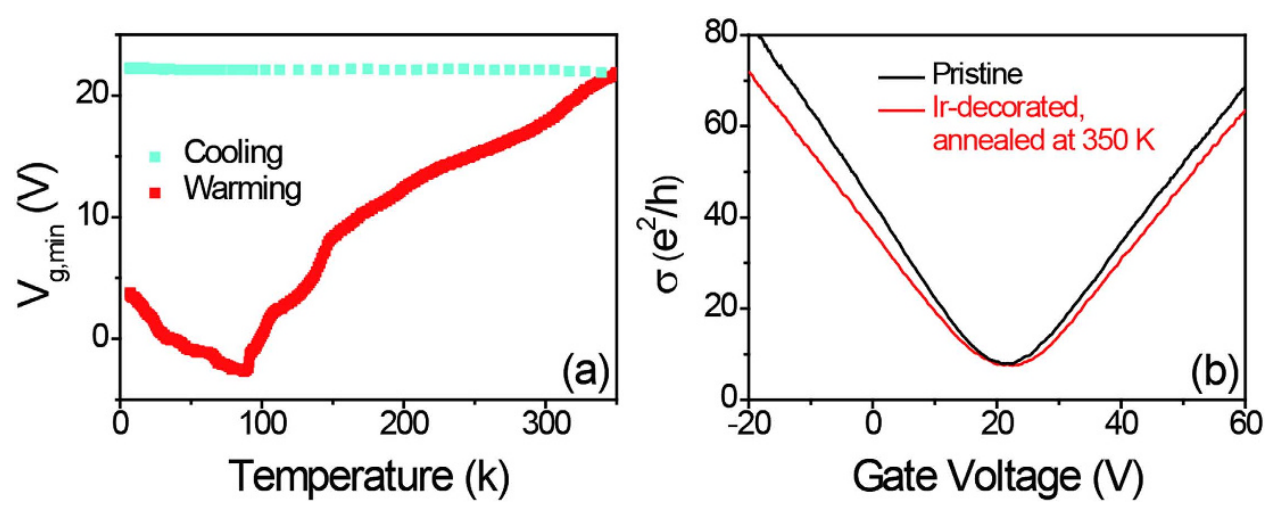

Figure 3. Temperature dependence of $V_{g, \min }$ and $\sigma\left(V_{g}\right)$. (a) $V_{g, \min }$ of graphene decorated with $0.085 \mathrm{ML}$ Ir as a function of temperature. (b) A comparison of $\sigma\left(V_{g}\right)$ for pristine graphene and for 0.085 ML Ir-decorated graphene deposited at $7 \mathrm{~K}$ and annealed at $350 \mathrm{~K}$. Data was taken at $7 \mathrm{~K}$.

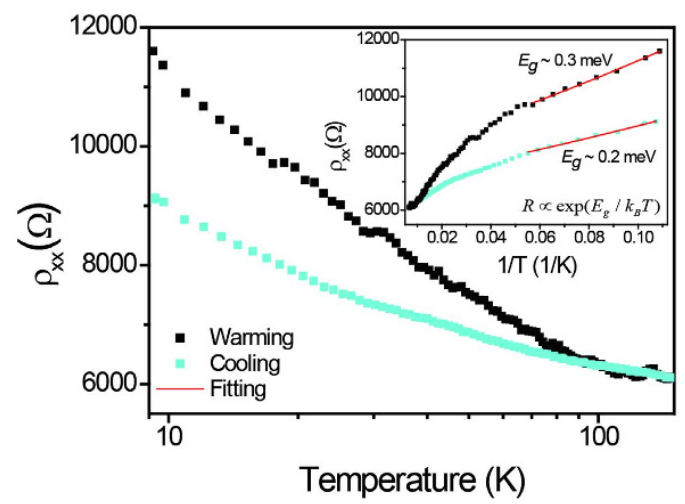

Figure 4. Temperature dependence of $\rho_{\mathrm{xx}}$ at $V_{\mathrm{g}}=V_{\mathrm{g}, \mathrm{min}}$ for $0.4 \mathrm{ML}$ Ir-decorated graphene. Data are shown for warming to $150 \mathrm{~K}$ after low temperature deposition, and subsequent cooling, as indicated in legend. Inset: Temperature dependence of $\rho_{\mathrm{xx}}$ at $V_{\mathrm{g}}=V_{g, \min }$ for $0.4 \mathrm{ML}$ Ir-decorated graphene. The red lines are fits to the thermal activation model as described in the text.

re-cooling to low temperature, i.e. the ripening process is irreversible, as shown in Fig. 3(a). Figure 3(b) shows a comparison of $\sigma\left(\mathrm{V}_{\mathrm{g}}\right)$ for pristine graphene and Ir-decorated graphene annealed to form large clusters. $V_{\mathrm{g} \text {,min }}$ remains almost the same, indicating there is no change in charge transfer between large Ir clusters and graphene, consistent with charged-impurity-dominated scattering, $\sigma_{\min }$ is nearly unchanged, and $\mu$ barely decreases, from 7000 to $6000 \mathrm{~cm}^{2} / \mathrm{Vs}$. In contrast to small clusters, large clusters have low impact on the conductivity of graphene. The results are similar to those seen with Au clusters ${ }^{4,7}$ on graphene.

We further studied the temperature dependence of $\rho_{\mathrm{xx}}$ of graphene decorated with Ir to search for an energy gap induced by spin-orbit coupling ${ }^{15}$. As shown in Fig. 4, $\rho_{\mathrm{xx}}$ increases with decreasing temperature, and is well described by $\rho_{\mathrm{xx}}(T) \sim \ln (T)$. Since the Ir cluster ripening process is irreversible when increasing and decreasing temperature (as shown in Fig. 3), the $\rho_{\mathrm{xx}}(T)$ curves of warming and cooling do not overlap. Comparing to the cooling curve, the Ir clusters rearrangement also provides additional contribution to the $\mathrm{T}$ dependence when warming. We also plot $\rho_{\mathrm{xx}}$ in logarithmic scale as a function of inverse temperature (inset of Fig. 4) and find $\rho_{\mathrm{xx}}(T)$ is poorly described by the simple thermal activation model $\rho_{x x} \propto e^{E_{g} / k_{B} T}$; The obtained fitting gap is extremely small, $E_{g}<1 \mathrm{meV}$, which is nonphysical, in that it is smaller than the measurement temperature $k_{\mathrm{B}} T$ and much smaller than the disorder energy scale of order $50 \mathrm{meV}$. The roughly logarithmic $\rho_{\mathrm{xx}}(T)$ may originate from increased weak localization in graphene, has been observed in other noble metal-decorated graphene ${ }^{24}$. The enhanced spin-orbit coupling in Ir-decorated graphene was also not seen by the non-local transport measurement, as discussed elsewhere ${ }^{25}$. We speculate that the failure to observe the predicted enhanced spin-orbit coupling and substantial energy gap in Ir-decorated graphene is because of the formation of Ir clusters on graphene, which is different from the single adatom model used in the theory ${ }^{15}$. Adatom clustering has also been shown to have a detrimental effect on the formation of the topological phase since the induced spin-orbit coupling vanishes in the region between the islands, which leads to the failure to observe the topological 
bulk gap ${ }^{26}$. The spin-orbit coupling induced energy gap was also not observed when the Ir coverage increases to as high as of $4.3 \mathrm{ML}$, this is consistent with the lack of any observed enhanced spin-orbit coupling in graphene grown on Ir (111) crystal $^{27}$. These results suggest the coupling between graphene and $\mathrm{Ir}$ is strongly dependent on the existing form of Ir.

In summary, we have investigated the electronic transport properties of graphene decorated with $5 d$ transitional metal Ir. Ir tends to form clusters on graphene, acting as charged impurity scattering centers with a single electronic charge per cluster. No topological gap induced by spin-orbit coupling is observed, either due to the lack of such a gap in graphene with clustered Ir, or the lack of a global gap in transport due to inhomogeneity in graphene with adatom clusters. These findings provide guidance for future experiments aimed at achieving strong spin-orbit coupling in metal-decorated graphene.

\section{Methods}

Graphene devices fabrication and Electrical transport measurements. Graphene flakes are obtained by mechanical exfoliation of graphite on a $300-\mathrm{nm}-\mathrm{SiO}_{2} / \mathrm{Si}$ substrate and are identified by color contrast in optical microscope imaging and confirmed by Raman spectroscopy. The electrical contacts are defined with standard electron beam lithography and thermally evaporated $\mathrm{Cr} / \mathrm{Au}(5 \mathrm{~nm} / 100 \mathrm{~nm})$. After annealing in $\mathrm{H}_{2} / \mathrm{Ar}$ gas at $350^{\circ} \mathrm{C}$ to remove resist residue $\mathrm{e}^{28}$, the device was mounted on a cryostat in an UHV chamber. All measurements were taken by using a conventional four-probe lock-in technique with a low frequency of $3.7 \mathrm{~Hz}$.

\section{References}

1. Castro Neto, A. H., Guinea, F., Peres, N. M. R., Novoselov, K. S. \& Geim, A. K. The electronic properties of graphene. Rev. Mod. Phys. 81, 109-162 (2009).

2. Chen, J.-H., Li, L., Cullen, W. G., Williams, E. D. \& Fuhrer, M. S. Tunable Kondo effect in graphene with defects. Nat. Phys. 7, 535-538 (2011).

3. Chen, J. H. et al. Charged-impurity scattering in graphene. Nat. Phys. 4, 377-381 (2008).

4. McCreary, K. M. et al. Effect of cluster formation on graphene mobility. Phys. Rev. B 81, 115453 (2010).

5. Pi, K. et al. Electronic doping and scattering by transition metals on graphene. Phys. Rev. B 80, 075406 (2009).

6. Ren, Y. et al. Controlling the electrical transport properties of graphene by in situ metal deposition. Appl. Phys. Lett. 97, 053107 (2010).

7. Wu, Y. et al. Tuning the doping type and level of graphene with different gold configurations. Small 8, 3129-3136 (2012).

8. Jeong, H. Y. et al. Flexible room-temperature $\mathrm{NO}_{2}$ gas sensors based on carbon nanotubes/reduced graphene hybrid films. Appl. Phys. Lett. 96, 213105 (2010).

9. Jang, C. et al. Tuning the Effective Fine Structure Constant in Graphene: Opposing Effects of Dielectric Screening on Short- and Long-Range Potential Scattering. Phys. Rev. Lett. 101, 146805 (2008).

10. Wang, Y., Bao, W., Xiao, S., Fuhrer, M. S. \& Reutt-Robey, J. Electrical detection of CF3Cl phase transitions on graphene. Appl. Phys. Lett. 103, 201606 (2013).

11. Elias, D. C. et al. Control of Graphene's Properties by Reversible Hydrogenation: Evidence for Graphane. Science 323, 610-613 (2009).

12. Cheng, S. H. et al. Reversible fluorination of graphene: Evidence of a two-dimensional wide bandgap semiconductor. Phys. Rev. B 81, 205435 (2010).

13. Weeks, C., Hu, J., Alicea, J., Franz, M. \& Wu, R. Engineering a Robust Quantum Spin Hall State in Graphene via Adatom Deposition. Phys. Rev. X 1, 021001 (2011).

14. Zhang, H., Lazo, C., Blügel, S., Heinze, S. \& Mokrousov, Y. Electrically Tunable Quantum Anomalous Hall Effect in Graphene Decorated by $\$ 5 \mathrm{~d} \$$ Transition-Metal Adatoms. Phys. Rev. Lett. 108, 056802 (2012).

15. Hu, J., Alicea, J., Wu, R. \& Franz, M. Giant Topological Insulator Gap in Graphene with \$5d\$ Adatoms. Phys. Rev. Lett. 109, 266801 (2012).

16. Qin, Y. et al. Enhanced quantum coherence in graphene caused by Pd cluster deposition. Appl. Phys. Lett. 106, 023108 (2015).

17. Jia, Z. et al. Transport study of graphene adsorbed with indium adatoms. Phys. Rev. B 91, 085411 (2015).

18. Straßer, C. et al. Long- versus Short-Range Scattering in Doped Epitaxial Graphene. Nano Lett. 15, 2825-2829 (2015).

19. Chandni, U., Henriksen, E. A. \& Eisenstein, J. P. Transport in indium-decorated graphene. Phys. Rev. B 91, 245402 (2015).

20. Adam, S., Hwang, E. H., Galitski, V. M. \& Das Sarma, S. A self-consistent theory for graphene transport. Proc. Natl. Acad. Sci. USA 104, 18392-18397 (2007).

21. Novikov, D. S. Numbers of donors and acceptors from transport measurements in graphene. Appl. Phys. Lett. 91, 102102 (2007).

22. N’Diaye, A. T., Bleikamp, S., Feibelman, P. J. \& Michely, T. Two-Dimensional Ir Cluster Lattice on a Graphene Moiré on $\operatorname{Ir}(111)$. Phys. Rev. Lett. 97, 215501 (2006).

23. Nakada, K. \& Ishii, A. DFT Calculation for Adatom Adsorption on Graphene. Graphene Simulation (InTech, Rijeka, 2011)

24. Li, W. et al. Electron localization in metal-decorated graphene. Phys. Rev. B 84, 045431 (2011).

25. Wang, Y., Cai, X., Reutt-Robey, J. \& Fuhrer, M. S. Neutral-current Hall effects in disordered graphene. arXiv:1504.05785 (2015).

26. Cresti, A., Van Tuan, D., Soriano, D., Cummings, A. W. \& Roche, S. Multiple Quantum Phases in Graphene with Enhanced Spin-Orbit Coupling: From the Quantum Spin Hall Regime to the Spin Hall Effect and a Robust Metallic State. Phys. Rev. Lett. 113, 246603 (2014).

27. Calleja, F. et al. Spatial variation of a giant spin-orbit effect induces electron confinement in graphene on $\mathrm{Pb}$ islands. Nat. Phys. 11, 43-47 (2015)

28. Ishigami, M., Chen, J. H., Cullen, W. G., Fuhrer, M. S. \& Williams, E. D. Atomic Structure of Graphene on $\mathrm{SiO}_{2}$. Nano Lett. 7, 1643-1648 (2007).

\section{Acknowledgements}

This work was supported by the NSF-MRSEC at the University of Maryland, DMR 0520471. M.S.F. is supported by the Australian Research Council. 


\section{Author Contributions}

Y.W., M.S.F. and J.R.R. conceived the experiments. Y.W., X.C. and W.B. fabricated graphene devices. Y.W. and S.X. performed the measurements. Y.W., M.S.F. and J.R.R. analyzed the data, discussed the results and wrote the manuscript.

\section{Additional Information}

Competing financial interests: The authors declare no competing financial interests.

How to cite this article: Wang, Y. et al. Electronic transport properties of Ir-decorated graphene. Sci. Rep. 5, 15764; doi: 10.1038/srep15764 (2015).

(c) (i) This work is licensed under a Creative Commons Attribution 4.0 International License. The images or other third party material in this article are included in the article's Creative Commons license, unless indicated otherwise in the credit line; if the material is not included under the Creative Commons license, users will need to obtain permission from the license holder to reproduce the material. To view a copy of this license, visit http://creativecommons.org/licenses/by/4.0/ 